\title{
Thermo-mechanical and Mold Flow Analyses of Die Shift in Wafer Reconstitution Process for Advanced Packaging Technology
}

\author{
Cheng-Ying Yang ${ }^{\text {a,*}}$, Yu-Cheng Liu ${ }^{\mathrm{a}}$, Kuo-Shen Chen ${ }^{\mathrm{a}}$, Tian-Shiang Yang ${ }^{\mathrm{a}}$, and Yao-Chen Wang ${ }^{\mathrm{b}}$ \\ ${ }^{a}$ Department of Mechanical Engineering, National Cheng Kung University, Tainan 70101, Taiwan \\ ${ }^{\mathrm{b}}$ Advanced Semiconductor Engineering (ASE), Co. Ltd, Kaohsiung 81170, Taiwan \\ *Corresponding Author: st936104@gmail.com
}

\begin{abstract}
In advanced wafer level packaging, a process called wafer reconstitution was developed as a strategy for decoupling IC/MEMS fabrication and packaging. However, several concern such as die-shift and warpage are found during reconstitution and they induce problems for subsequent wafer process. In this paper, it is desired to examine the key factor of die-shift on wafer. The investigation procedures including fluidic and thermomechanical effects are introduced. Preliminarily, the die-shift problem is deduced as interaction of fluid load, thermal expansion, shrinkage of molding compound and viscoelastic effect. To gain a deeper insight, simplified fluid model and finite element analyses have been constructed to mimic the entire process. As current results shown, defects of molding is not the dominating factor in reconstitution process as those usually reported previously. Meanwhile, simulation of thermal model has a consistent tendency with actual situation and is close to the observed defects. In summary, the presented model in this work is able to provide the basis failure phenomena for the wafer reconstitution. However, the situations are actually sensitive to processing parameters and a more sophisticated finite element model to include those detail processing should be further developed for further optimizing the reconstitution process for a better yield models in the near future.
\end{abstract}

Keywords: wafer reconstitution, die-shift, Finite element analysis, Mold flow analysis

\section{Introduction}

Recently, with the advanced in semiconductor fabrication technology, the characterization dimensions of IC devices have been reduced rapidly. On the other hand, for the field of electronic packaging, although the development is also rapid, it still cannot match the advancement in both IC and MEMS technologies. As a result, an additional process was developed to allow the usage of existed packaging equipment on far more advanced IC chips. Such a process is called as wafer reconstitution (Recon). As shown in Figure 1 , the Recon process is initiated in placing die-sawed chips on a tape attached on a stainless steel carrier with a predesignated pattern and spacing for subsequent packaging processing equipment. Followed by a molding process to injecting compounds to form a die-compound-carrier wafer. Such a 12 " wafer is then experienced a series of thermal processing before removing the carrier and slicing off extra compounds. Finally, the compound wafer is diced for individual packaging. By such an effort, the development in IC/MEMS processing and IC/MEMS packaging can be fully decoupled. However, such a process introduces numerous mechanics and thermos-mechanical concerns. For example, as shown in Figure 2, the fluid loading during molding could possibly cause separation between dies and the tape or the permanent deformation of the tape underneath (i.e., fly die problem). The thermal stress generated during curing due to mismatch of coefficient of thermal expansion coefficients between major components, as well as the possible shrinkage and stress relaxation of molding compounds, could also contribute the deformation and warpage of the compound wafer. The in-plane deformation is called as die shift, in which the die is not swept completely away from their original position. Instead, a slight shift in position is observed. This slight shift in die location creates problems for subsequent wafer processing, such as the formation of redistribution lines. Previous studies indicated that both fluid loading and thermal mechanical mismatch during curing play key roles in this problem ${ }^{(1)}$.However, under different molding and curing conditions, as well as the used materials, the major controlling factor could be different case by case.

In this work, die shift approximately $30 \mu \mathrm{m}$ was reported 
on Recon process. It is desired to find the major controlling factor to minimize or pre-compensate the die shift effect and this work addresses the investigation procedure and presents the current results and conclusion as the basis for process optimization.

The remaining of this article mainly addresses the above investigation in detail. In Section II, the overall investigation procedure is outlined in detail for introducing the entire problems in Recon process, as well as the corresponding modeling tasks. Essential fluid mechanics analyses for molding process is then performed in Section III for quantifying the influence of the fluid dragging force. In parallel, several finite element (FE) thermal stress analysis models are presented in Section IV for mimicking the curing process and for evaluating the effect of thermal stress on the die shift. Finally, an overall discussion is addressed in Section $\mathrm{V}$ for commenting the current investigation and presenting the future direction for improving the die shifting problem and followed by an overall conclusion in Section VI.

(a)

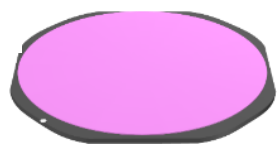

(c)

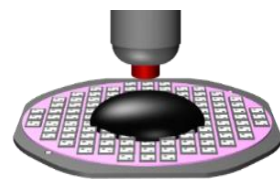

(b)

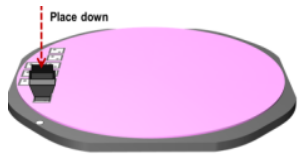

(d)

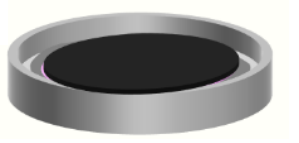

(e)

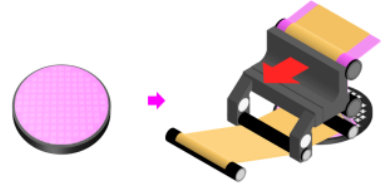

(f)

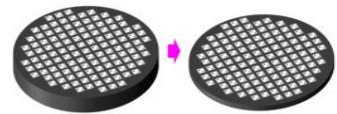

Fig. 1. The schematic flow of the wafer Recon process. (a) deploying a tape on a carrier, (b) die attachment, (c) molding, (d) curing, (e) separation of carrier and tape, (f) wafer slicing.

(a)

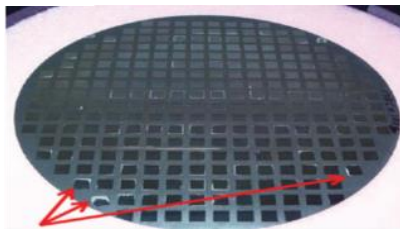

(b)

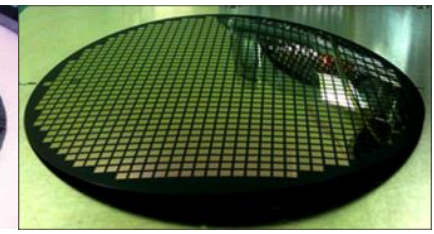

Fig. 2. Common failures observed in Recon process. (a) die-shift and fly die (indicated by red arrows) and (b) excessive wafer warpage [2].

\section{Overall analyses procedure}

\subsection{Investigation flow set-up}

The investigation flow is shown in Figure 3. There are three major investigations performed in parallel. First, an analytical model on molding process is established based on elementary fluid mechanics for predicting the possible die shifting due to fluid loading. On the other hand, several FE models are also constructed to mimic the entire Recon process after molding (such as heating, curing, cooling, holding temperature and carrier removal) for evaluating the possible thermal deformation. Finally, essential material characterizations are conducted for evaluating the material properties (modulus, yielding strength, and viscoelastic properties, and adhesion strength) of both molding compounds and tapes in order to support the above two analyses.

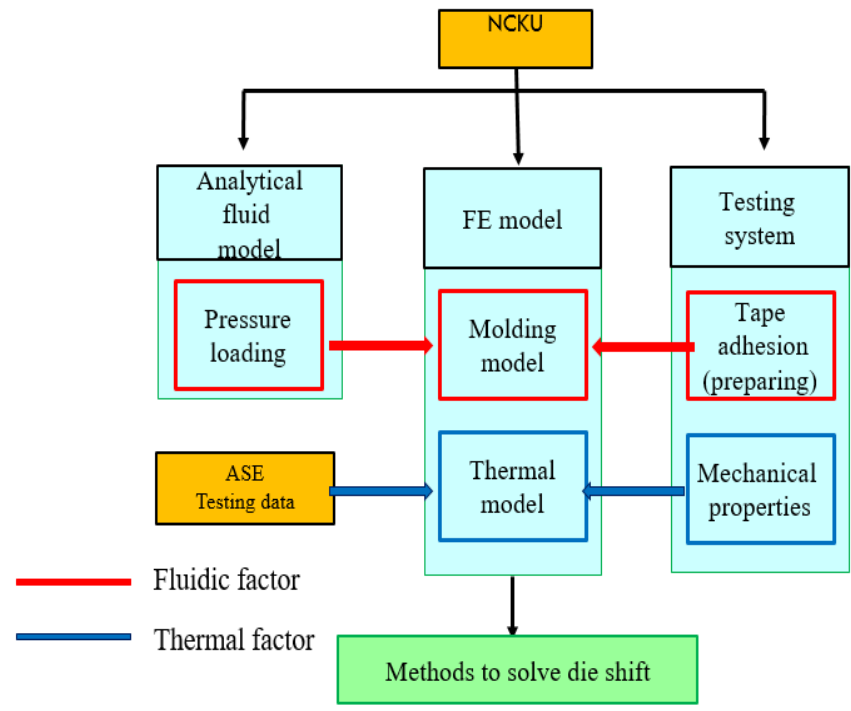

Fig. 3. Investigation flow for addressing die-shift problem

\subsection{Major analysis tasks}

In this work, the main stress falls on the processes of molding, post mold cure and debonding which are highly correlated with the observed die shift phenomenon. The factors causing defects in three procedures could be further divided into two main analysis categories, mechanical or fluidic effects.

In fluidic aspect, the strength of tape is believed to be the core problem due to effect of mold-flow. We believe that two cases should be considered. First, the possible yielding 
may occur in tape due to combined loading of mold-flow. And second, under such a significant pressure gradient during molding, the adhesive ability of tape could be questionable. In both of the cases, die-shift problem could be treated as the result from the tape failure.

In mechanical aspect, key failure sources could be induced by several mechanisms. First, the thermal expansion and CTE mismatch in materials. Second, the residual stress generated due to geometry constraints of carrier. Third, shrinkage during curing and stress relaxation behaviors of molding compounds. The preliminary analysis results indicate that the thermal stress during curing is the current controlling factor. On the other hand, the die shift due to fluid loading during molding seems not significant so far. However, this conclusion could be changed if a different molding temperature is chosen since the tape strength (providing the resistivity) and compound viscosity (providing drag force) are strong functions of temperature.

\section{Mold flow analysis}

\subsection{Molding analysis introduction}

Previously, during the early stage in Recon process development, several research works have performed to evaluate the effect of molding flow on die-shift ${ }^{(2,3)}$. In the work, the effective load on die caused by mold-flow is also estimated by an analytical model. This model showed that the pressure gradually decreased from the center. Meanwhile, the dies at outer region were subjected to higher shear stress than that at the central regime. Since the strength of tape is a key factor for preventing die-shift. Therefore, a material with higher yield strength or elastic modulus is preferred for decreasing the failure probability ${ }^{(4)}$. Consequently, the variation in yield strength of a tape resulting from temperature change should also be considered. Here, an analytical mold flow model is established to calculate the pressure distribution. Based on the characterized properties, the resulted die shift can be estimated. In parallel, a FE model is also constructed to estimate the die shift by considering possible material yielding for evaluating the relative significance of molding issues in die shifting.

\subsection{Fluid mechanics analysis}

The molding compound used could be treated as a viscous fluid before curing. Starting from the low-Reynolds number squeeze flow analysis, the momentum equations of Newtonian and power-law fluid could be simplified by lubrication theory ${ }^{(5,6)}$. In conjunction with the law of mass conservation, one could obtain the relationship between molding velocity and flow-field pressure.

For simplicity, two dimension axisymmetric model was used to estimate the pressure distribution shown in Figure 4(a). The momentum equation can be expressed as

$$
\rho\left\{\frac{\partial V}{\partial t}+(V \cdot \nabla) V\right\}=-\nabla P+\eta \nabla(\nabla \cdot V)+\rho f,
$$

where $\rho$ is density, $V$ the fluid velocity, $P$ the pressure, $\eta$ the viscosity, and $f$ the gravity effect.

In this analysis, the model could be further simplified by the following assumptions: (I) Newtonian fluid; (II) body force is negligible; (III) very low Reynolds number; (IV) small film thickness; and (V) flow primarily in x-dir. Then Eq. (1) can be simplified as

$$
\frac{\partial P}{\partial x}=\eta \frac{\partial^{2} u}{\partial z^{2}}
$$

With no slip condition, fluid velocity and flow rate could be obtained. By giving the molding velocity $V$, and followed by conservation of mass. Eq. (2) becomes as

$$
-\frac{h^{3}}{12 \eta} \frac{\partial P}{\partial x}=-x V .
$$

Then the simplified model could be extended into a model with $\mathrm{n}$ chips deployed in $\mathrm{x}$-direction with dimensions shown in Figure 4(b). And it is possible to link the resulted shear stress acting on dies and the molding speed $V$.

$$
\begin{gathered}
P_{i}=\frac{6 \eta V}{h_{i}^{3}}\left(x^{2}-L_{i}^{2}\right)+\sum_{j=1}^{n+1-i} \frac{6 \eta V}{h^{3}{ }_{i+j}}\left(L_{i+j-1}^{2}-L_{i+j}^{2}\right) \\
|\tau|=\frac{12 \eta V}{h^{3}} \frac{h}{2} x=\frac{6 \eta V x}{h^{2}}
\end{gathered}
$$

The pressure distribution result is shown in Figure 4(c) and will be used for subsequent FE simulation for estimating the amount of flow-induced die shift. 


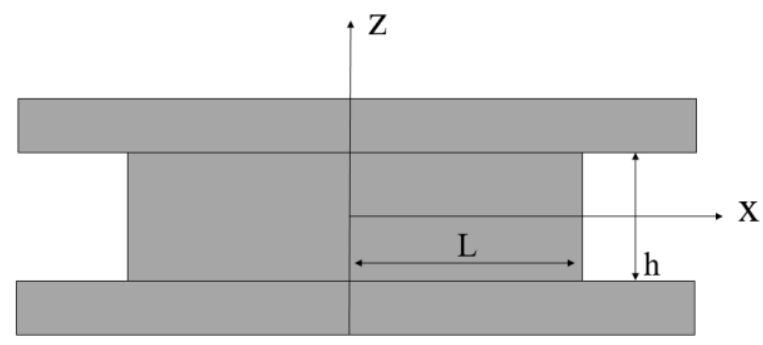

(a) Schematic diagram of 2D simplified model.

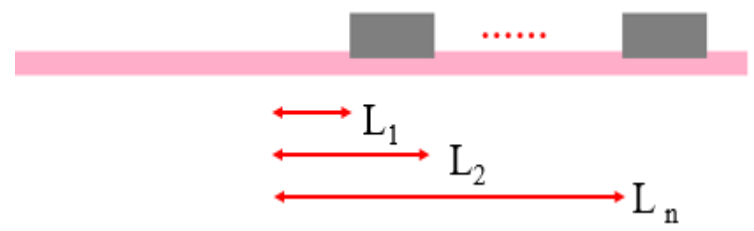

(b) Analytic molding model having $\mathrm{n}$ dies.

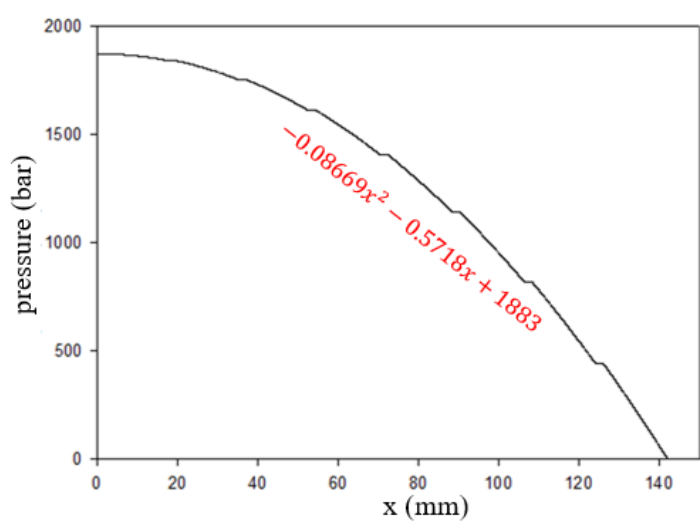

(c) Pressure distribution in molding process.

Fig. 4. Analytic molding model result.

\subsection{Tape property test}

The elastic modulus and strength of tape are critical factors in determining die shift level during molding and should be characterized. In order to gain the information for FE analysis, an uniaxial tensile test facility with temperature controlled is conducted and is shown in Figure 5(a)(b). With the controlled temperature chamber, the properties of the tape at different temperatures was obtained. Figure 5(c) is the stress-strain curve at different temperatures. It shows that both the Young's modulus and yield strength decrease with temperature. The obtained temperature-dependent material properties are then included into the FE models for performing simulations in thermo-mechanical.

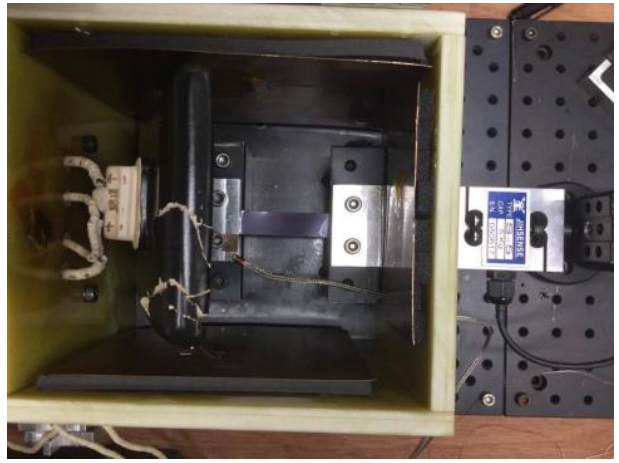

(a) Testing environment for obtaining tape properties

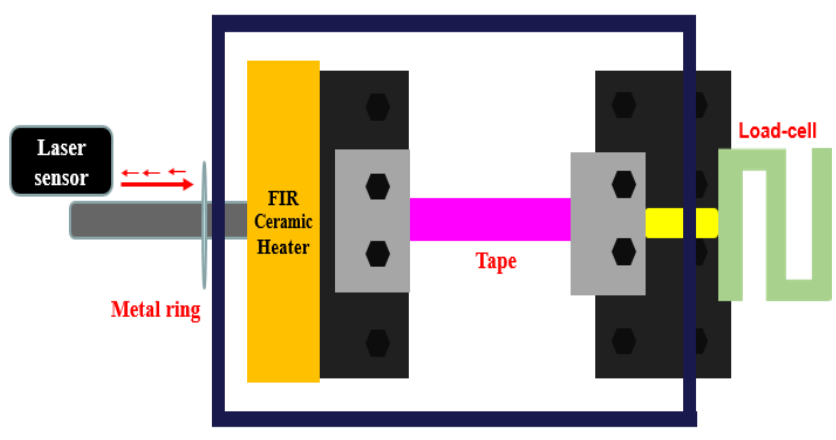

(b) Testing environment schematic diagram.

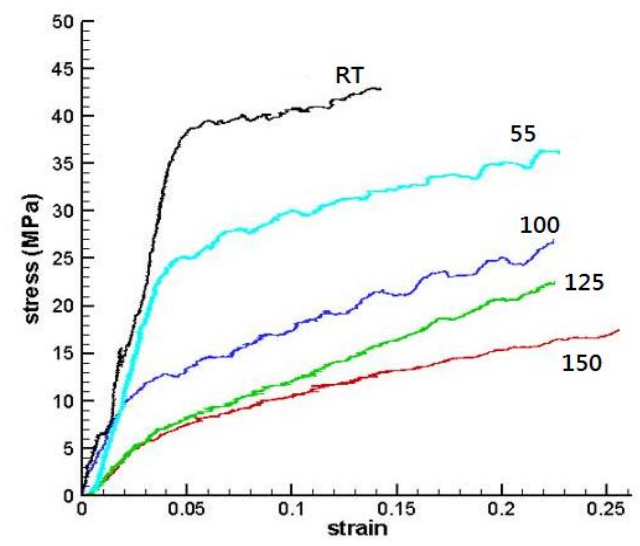

(c) Stress-strain curves under different temperatures.

Fig. 5. Tape testing system and results

\subsection{Finite element analysis of molding with tape}

The Simulia Abaqus is used as the tool for performing the FE analysis. The FE model used in molding process is constructed with C3D20 3D continuum elements with material parameters listed in Table 1. In molding process, dies are subjected to distributed pressure loading at $125^{\circ} \mathrm{C}$ as schematically shown in Figure 6(a). To simplify the analysis, the model contains only a fully attached die at the critical location. The calculated deformation is shown in Figure 6(b). For example, die shift approximately $5 \mu \mathrm{m}$ is observed due to the stretching in tape at $125^{\circ} \mathrm{C}$. The shift become worsen for 
further increasing in molding temperature shown in Figure 6(c) since the tape strength drops dramatically with temperature.

Since the amount of die-shift due to molding is much less than the observed results $(30 \mu \mathrm{m})$, it could conclude that molding is not the current key factor in die-shift. Nevertheless, it shows that the fluid loading effect may not be completely negligible especially for further increasing in molding temperature.

Table 1. Material properties under $125^{\circ} \mathrm{C}$

\begin{tabular}{|c|c|c|c|c|c|}
\hline & $\begin{array}{c}\text { Element } \\
\text { type }\end{array}$ & $\begin{array}{c}\text { No. of } \\
\text { elements }\end{array}$ & $\begin{array}{c}\text { Young's } \\
\text { modulus }\end{array}$ & $\begin{array}{c}\text { Poisson's } \\
\text { ratio }\end{array}$ & $\begin{array}{c}\text { Yield } \\
\text { strengh }\end{array}$ \\
\hline Die & C3D20 & 45 & $120 \mathrm{GPa}$ & 0.25 & \\
\hline Tape & C3D20 & 225 & $0.19 \mathrm{GPa}$ & 0.4 & $7.75 \mathrm{MPa}$ \\
\hline
\end{tabular}

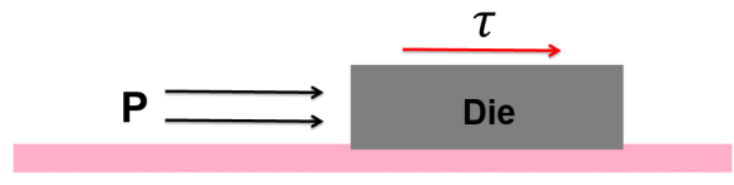

(a) Schematic diagram of molding model

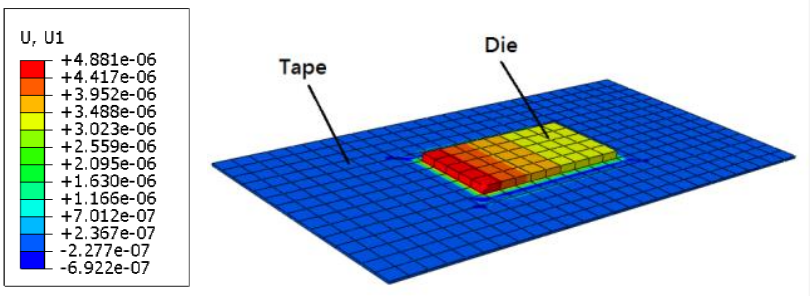

(b) FE result of die-shift in molding.

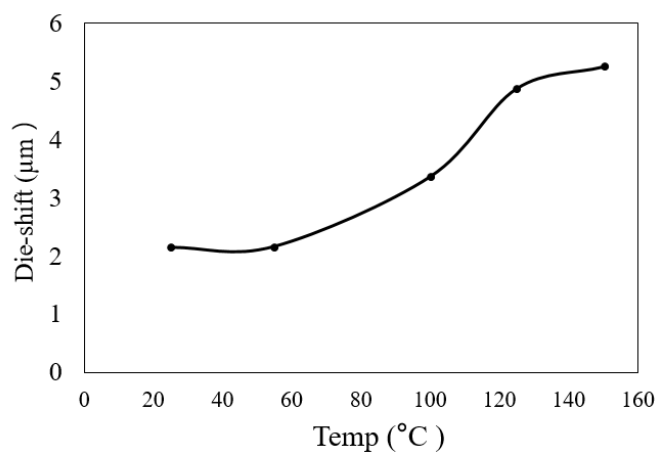

(c) Die-shift versus molding temp. in FE model.

Fig. 6. FE results in molding process

\section{Thermo-mechanical analysis}

\subsection{Thermal analysis introduction}

After molding process, the wafer is then experienced a series of varying temperature treatments. The major two processes, post mold cure and debonding, are discussed in this section. The post mold cure process is aimed for accelerating the curing reaction time of molding compound in order to stabilize the compound properties for further reducing defects. However, the induced die-shift is inevitable. In thermo-mechanical part, thermal properties in materials play key roles. The induced CTE mismatch between the molding compound, tape and carrier could cause great warpage ${ }^{(7)}$. In previous research ${ }^{(8)}$, it was found that using different CTE materials as wafer carrier resulted in significant difference in die-shift level. Furthermore, thermal expansion and cure shrinkage, as well as stress relaxation in compound, will induce residual stresses due to the geometry constraints from the carrier. Once the carrier is removed, the accumulating stress at the material interface will be released and this could cause significant die-shift. In the following sections, several FE thermal analysis modules have been set up based on the thermal-mechanical properties for assessing the major die-shift factors.

\subsection{FE model construction}

Two types of models are introduced, as Figure 7 shown. A two dimensional model is firstly constructed for effectively performing the analyses. In this $2 \mathrm{D}$ simulation, the tape is absent from the model for cost-effective consideration. The model is constructed using quadratic axisymmetric element (CAX8). Subsequently, a quarterwafer three-dimensional model with tetrahedral element (C3D10) is also constructed for performing a far more detailed estimation. Here the tape is included with temperature dependent properties.

In both models, the thermos-mechanical properties of the compound, such as curing shrinkage and stress relaxation, are also included. However, dies in 2D axisymmetric model tends to over-estimated the wafer stiffness due to its modeling limitation shown in Figure 8. Therefore, the 3D model within the actual layout of die attachment is used to emulate the overall Recon procedure. 


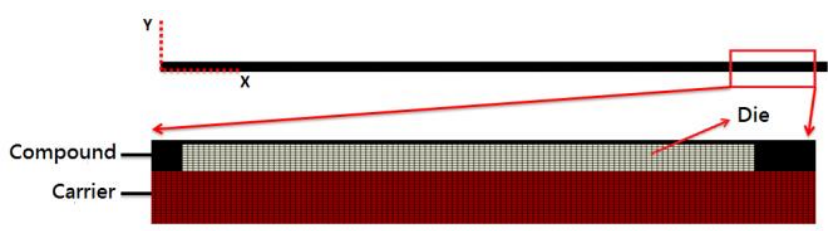

(a) Partial enlargement of 2D axisymmetric model.

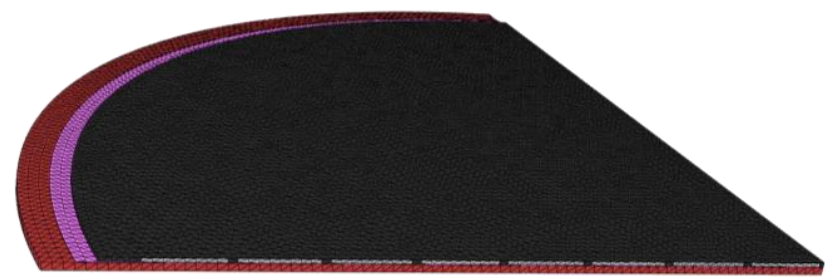

(b) 3D model with tape included.

Fig. 7. Models in thermal analysis.

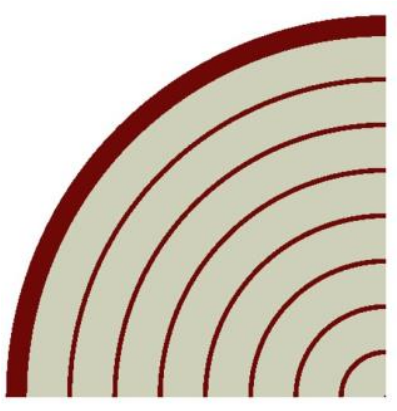

(a) $2 \mathrm{D}$ axisymmetric model

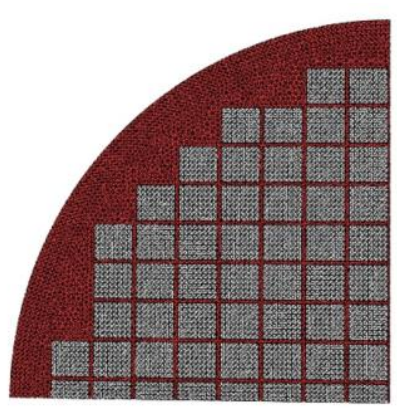

(b) $3 \mathrm{D}$ model
Fig. 8. Difference in die between 2D and 3D FE model.

\subsection{FE process emulation}

The 2D model is used for developing individual process emulation with material parameters listed in Table 2. For addressing the effect of post mold cure, wafer is heated up to specified temperature and the CTE mismatch in composite structure induced thermal stress is shown in Figure 9(a).

Table 2. Material properties in 2D model

\begin{tabular}{|c|c|c|c|c|c|}
\hline & $\begin{array}{c}\text { Element } \\
\text { type }\end{array}$ & $\begin{array}{c}\text { No. of } \\
\text { elements }\end{array}$ & $\begin{array}{c}\text { Young's } \\
\text { modulus }\end{array}$ & $\begin{array}{c}\text { Poisson's } \\
\text { ratio }\end{array}$ & CTE \\
\hline Die & CAX8 & 8480 & $120 \mathrm{GPa}$ & 0.25 & $2.8\left(10^{-6} / \mathrm{C}\right)$ \\
\hline Compound & CAX8 & 3250 & $20 \mathrm{GPa}$ & 0.4 & $30\left(10^{-6} / \mathrm{C}\right)$ \\
\hline Carrier & CAX8 & 25000 & $210 \mathrm{GPa}$ & 0.3 & $12\left(10^{-6} / \mathrm{C}\right)$ \\
\hline
\end{tabular}

After heating up, the wafer is then held at the temperature for several hours. Due to the nature of polymers at elevated temperature, it is necessary to consider the stress relaxation characteristic in molding compound. Here, such a viscoelastic behavior is modeled as a generalized Maxwell fluid using Prony series. Using this preliminary model, the stress and deformation after several hours holding is shown in Figure 9(b).
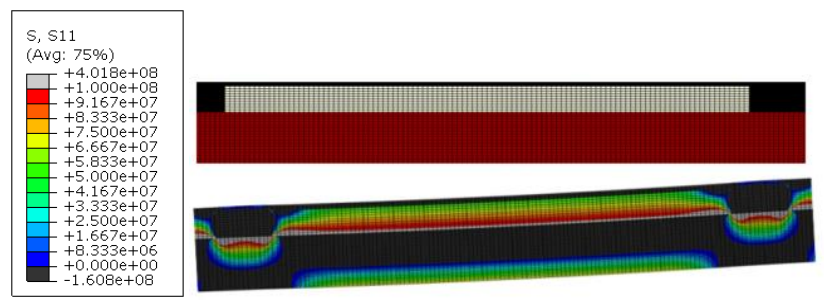

(a) Thermal stress in $\mathrm{x}$ direction of being heated to specified temp.
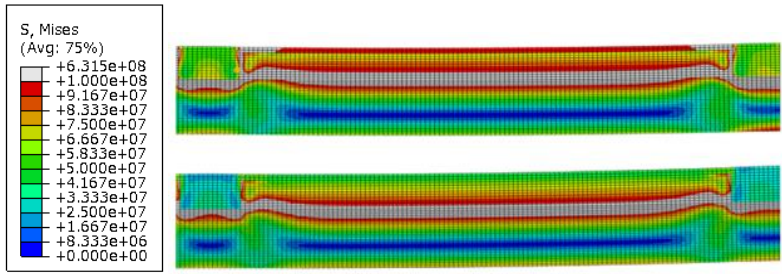

(b) Viscoelastic effect in molding compound of holding temp for several hours.

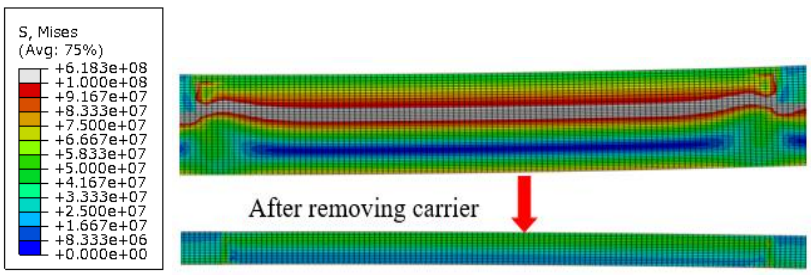

(c) Residual stress release due to carrier removal (debonding)

Fig. 9. Important analysis results in 2D model

The post mold cure process associated with crosslinking reaction cause shrinkage in compound, thus producing possible die-shift ${ }^{(9,10)}$. The volume shrinkage rate is on the order of approximately $2.4 \%$. In this preliminary model, an initial tensile stress is given into compound to mimic the shrinkage effect. The combined volume change between thermal expansion and shrinkage is also constrained by the stainless steel carrier and this induces extra residual stress at the interface. Once the carrier is removed, a significant reduction in both the die-shift and the warpage levels are observed as shown in Figure 9(c).

\subsection{FE overall emulation}

The overall process emulation is based on the $3 \mathrm{D}$ model in conjunction with the temperature dependent material parameters plotted in Figure 10. Both the elastic modulus and yield strength of tape decrease with the temperature. On the other hand, elastic modulus of the compound slightly increases at higher temperature and its CTE changes dramatically from $7\left(\mathrm{ppm} /{ }^{\circ} \mathrm{C}\right)$ to $33\left(\mathrm{ppm} /{ }^{\circ} \mathrm{C}\right)$. The overall temperature history of emulation is illustrated in Figure 11(a), two steps are involved with time effect and the stress 
relaxation effect is considered. An initial stress is added into compound for representing the curing shrinkage behavior. The die-shifts caused by individual step is shown in Figure 11(b). In which the tendency is consistent with the observation on production line, and the preliminary simulation result $27 \mu \mathrm{m}$ is close to the observed defects approximately $30 \mu \mathrm{m}$ in die-shift report.

(a)

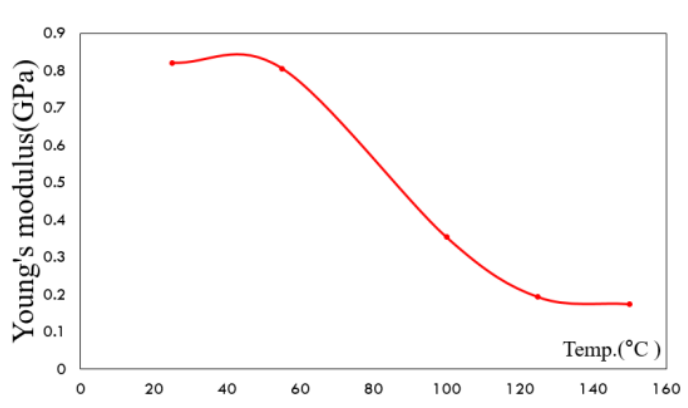

(b)

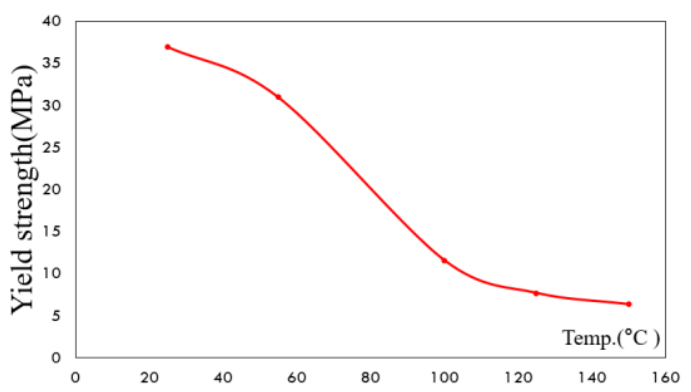

(c)

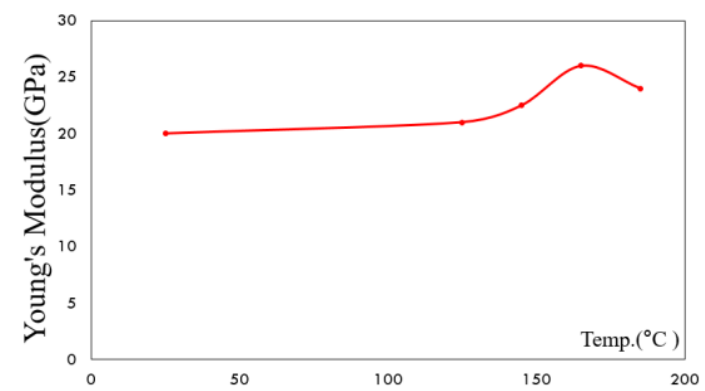

(d)

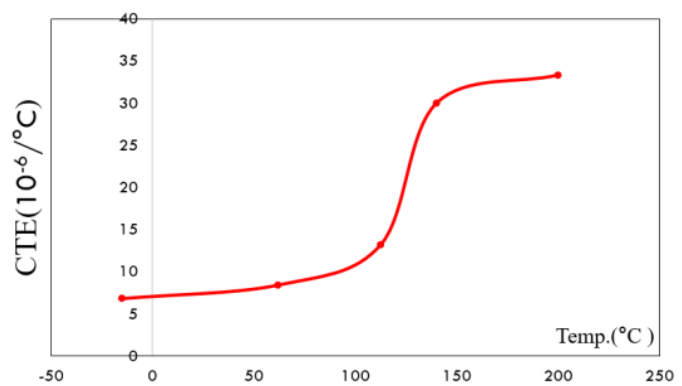

Fig. 10. Temperature dependent material parameter curves:

(a) Elastic modulus and (b) Yield strength of tape; (c)

Elastic modulus and (d) CTE of molding compound

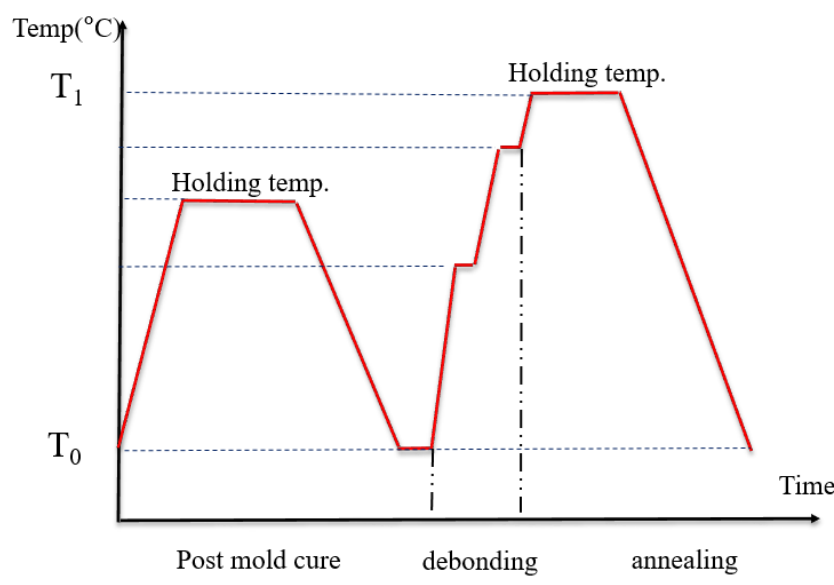

(a) The overall temperature history in thermal mechanical part, where $\mathrm{T}_{0}$ and $\mathrm{T}_{1}$ are in the range of $25^{\circ} \mathrm{C}$ to $250^{\circ} \mathrm{C}$

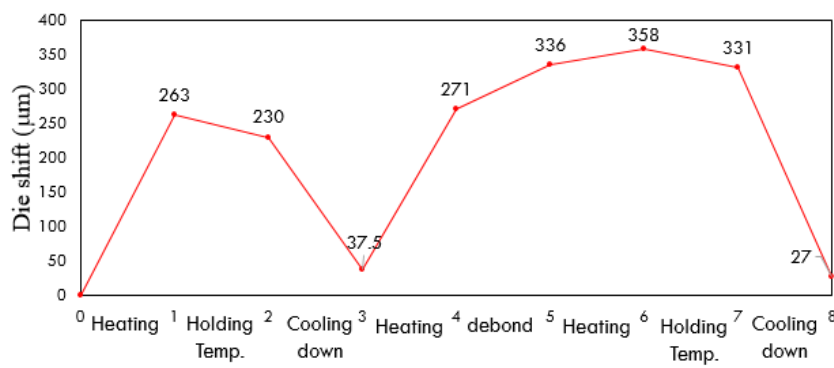

(b) The individual step result in FE analysis.

Fig. 11. The overall analysis procedure and result

Although this overall emulation model is still primitive, the agreement between simulation and experimental results provide a strong confidence on the feasibility of the simulation. In the future, more sophisticated model modifications will be added for allowing a more precisely prediction of die-shift and for developing processing optimization for reducing failure.

\section{Discussion}

The current work shows that molding process is not the major controlling factor for causing die shift if the tape having enough adhesive ability against mold-flow. Since adhesive ability might become the key factor of molding, the adhesive test under drag force should also be conducted. If the adhesion is sufficient strong enough against even higher loading, the molding temperature could be reduced slightly to improve the tape strength although the loading is also increased due to a higher fluid viscosity. Meanwhile, in thermo-mechanical analysis, the die-shift predicted by the 
fully viscoelastic and the linear elastic model is approximately $30 \mu \mathrm{m}$. Thus, more accurate viscoelastic properties should be obtained. Meanwhile, the preliminary shrinkage effect is currently mimicked by using a wide range of initial stress. In the future, Abaqus User-Subroutine UEXPAN will be used to describe the cure shrinkage in compound for a more adequate modeling.

To sum up, for the die-shift induced in our Recon process, the molding process seems not the major controlling factor but the adhesive ability of tape is still required to be confirmed. Meanwhile, a further characterization on temperature and time dependent material properties of the associated materials should be completed for developing more accurate FE models for predicting the failure and for improving the process design.

\section{Conclusion}

Die-shift problem is a critical issue in advanced packaging technology. In this research, two major analyses, mold-flow and thermo-mechanical investigation procedures are performed. The FE models on individual process step have been developed for identifying the key factor in dieshift. As current results shown, defects of molding is not the dominating factor in Recon process. Regarding to thermal analyses, the die-shift is close to the defect report approximately $30 \mu \mathrm{m}$. Thus, the presented model in this work could provide the basis FE solution for parameter optimization in wafer reconstitution.

\section{Acknowledgment}

This research is supported by the Ministry of Science and Technology in Taiwan, ROC under the contact number 105-2218-E-006-018.

\section{References}

(1) H. S. Ling, B. Lin, Ch. S. Choong, S. D. V., Chai Tai Chong : "Comprehensive study on the interactions of multiple die shift mechanisms during wafer level molding of multichip-embedded wafer level packages", IEEE Trans. Compon. Packag. Manuf. Technol., Vol. 4, pp.1090-1098, 2014.

(2) C. H. Khong, A. Kumar, X. W. Zhang, G. Sharma, S. R. Vempati, K. Vaidyanathan, J. H.-S. Lau, D.-L. Kwong : "A novel method to predict die shift during compression molding in embedded wafer level package", Proc.
Electron. Compon. Technol. Conf., pp. 535-541, 2009.

(3) L. Bu, S. Ho, T. C. Dexter, X. Zhang : "Investigation on die shift issues in the 12 inch wafer level compression molding process", IEEE Trans. Compon. Packag. Manuf. Technol., Vol. 3, pp. 1647-1653, 2013.

(4) J. Mazuir, V. Olmeta, M. Yin, G. Pares, A. Planchais, K. Inal, M. Saadaoui : "Evaluation and optimization of dieshift in embedded wafer-level packaging by enhancing the adhesion strength of silicon chips to carrier wafer", in Proc. 13th Electronics Packaging Technology Conference, pp. 747-751, 2011.

(5) F. Shuler, S.G. Advani : "Transverse squeeze flow of concentrated aligned fibers in viscous fluids", J. NonNewtonian Fluid Mech., Vol. 65, pp.47-74, 1996.

(6) C. Ghnatios, F. Chinesta, C. Binetruy : "3D modeling of squeeze flows occurring in composite laminates", International Journal of Material Forming, Vol. 8, pp.73-83, 2015.

(7) G. Sharma, A. Kumar, V. S. Rao, S. W. Ho, V. Kripesh : "Solutions strategies for die shift problem in wafer level compression molding", IEEE Trans. Compon. Packag. Manuf. Technol., Vol. 1, pp. 502-509, 2011.

(8) S. C. Chong, C. H. Khong, K. L. C. Sing, D. H. S. W., C. T. W. Liang : "Process challenges and development of eWLP”, in Proc. 12th Electronics Packaging Technology Conference, pp. 527-531, 2010.

(9) M. R. Kamal, M. E. Ryan : "The behavior of thermosetting compounds in injection molding cavities", Polymer Engineering and Science, Vol. 20, pp. 859-867, 1980.

(10) D.G. Yang, K.M.B. Jansen, L.J. Ernst, G.Q. Zhang, W.D. van Drief, H.J.L. Bressers : "Modeling of cureinduced warpage of plastic Ic packages", in Proc. 5th. Int. Conf. on Thermal and Mechanical Simulation and Experiments in Micro-electronics and Micro-Systems, pp. 32-40, 2004.

(11) Abaqus theory manual, Version 6.10, Simulia, 2009. 